\title{
Spatial fluctuations of a surviving particle in the trapping reaction
}

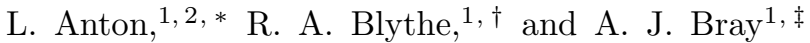 \\ ${ }^{1}$ Department of Physics and Astronomy, University of Manchester, M13 9PL, U.K. \\ ${ }^{2}$ Institute of Atomic Physics, INFLPR, Lab 22, PO Box MG-36 R76900, Bucharest, Romania
}

(Dated: November 9, 2018)

\begin{abstract}
We consider the trapping reaction, $A+B \rightarrow B$, where $A$ and $B$ particles have a diffusive dynamics characterized by diffusion constants $D_{A}$ and $D_{B}$. The interaction with $B$ particles can be formally incorporated in an effective dynamics for one $A$ particle as was recently shown by Bray et al. [Phys. Rev. E 67, 060102 (2003)]. We use this method to compute, in space dimension $d=1$, the asymptotic behaviour of the spatial fluctuation, $\left\langle z^{2}(t)\right\rangle^{1 / 2}$, for a surviving $A$ particle in the perturbative regime, $D_{A} / D_{B} \ll 1$, for the case of an initially uniform distribution of $B$ particles. We show that, for $t \gg 1,\left\langle z^{2}(t)\right\rangle^{1 / 2} \propto t^{\phi}$ with $\phi=1 / 4$. By contrast, the fluctuations of paths constrained to return to their starting point at time $t$ grow with the larger exponent $1 / 3$. Numerical tests are consistent with these predictions.
\end{abstract}

\section{INTRODUCTION}

Reaction-diffusion problems are a paradigm of irreversible nonequilibrium dynamics [1]. Among the conceptually simplest of such problems is the trapping reaction, $A+B \rightarrow B$, in which $A$ and $B$ particles diffuse in space. When an $A$ and $B$ particle meet the $A$ particle is annihilated, while there are no interactions between particles of the same species. Though simple to state, this problem is quite subtle: for example, the asymptotic behaviour of the $A$-particle density for one- and two-dimensional systems has only recently been computed [2, 3].

Recently there has been renewed interest [4, 5, 6, 7, 8, 9, 10, 11] in this reaction, stimulated in part by the advent of both new numerical methods [12] and new analytical techniques [2, 3]. The main focus of interest in this system has traditionally been the computation of the time-dependence of the $A$-particle density [13. Note that, since the $A$-particles are independent, this computation is equivalent to the computation of the survival probability, $Q(t)$, of a single $A$-particle moving in a sea of $B$ particles. In the case of one space dimension, to which this paper is devoted, $Q(t)$ is known to decay asymptotically as $\exp \left(-\lambda t^{1 / 2}\right)$ [13]. Recently, by a combination of bounding arguments, the constant $\lambda$ was determined exactly [2, 9]: $\lambda=(2 / \pi) \rho\left(4 \pi D_{B}\right)^{1 / 2}$, where $\rho$ and $D_{B}$ are the density and diffusion constant respectively of the $B$-particles. Note that the constant $\lambda$ does not depend on the $A$-particle diffusion constant, $D_{A}$, and the leading asymptotic result is the same as for a stationary $A$-particle.

A parallel advance in algorithmic methods 12] has enabled very long simulations of the model, especially in one dimension [3, 12], in which $Q(t)$ can be made arbitrarily small (of order $10^{-70}$, say, in reasonable computer time). Remarkably, the predicted asymptotic decay is not observed in simulations even for these very small values of $Q(t)$, motivating a study of the preasymptotic behaviour. In a companion paper [14], we have employed a path-integral approach [9] to compute, for small ratio $D_{A} / D_{B}$ of diffusion constants, the leading correction to the asymptotic form quoted above. Including this term gives reasonable agreement with data.

In the present paper we address, within the path-integral formalism, a different aspect of the problem. Specifically, we study the spatial fluctuations, $\left\langle x^{2}(t)\right\rangle$, of the $A$-particle averaged over the surviving trajectories at time $t$. We find that the fluctuations are subdiffusive: $\left\langle x^{2}(t)\right\rangle^{1 / 2} \sim t^{\phi}$ with $\phi<1 / 2$. In their numerical work, Mehra and Grassberger 12. find $\phi$ to be in the range $0.25-0.3$, where the effective exponent seems to be decreasing at larger $t$. We will argue that $\phi=1 / 4$.

Our approach is based on a recent development in ref. [9]. The main idea is to consider the probability of intersection before time $t, P(x, t ; z)$, between a $B$ particle starting from $x$ at $t=0$ and a fixed trajectory, $z(t)$, of the $A$ particle starting at $x=0$ when $t=0$. This probability can be averaged over the initial position of the $B$ particle (assumed uniform in length $V$, where we specialize to space dimension $d=1): R(t ; z) / V=(1 / V) \int d x P(x, t ; z)$. The survival probability, i.e. the probability of no collision up to time $t$, for the trajectory $z$ of the $A$ particle is $1-R(t ; z) / V$. If the system has $N B$-particles, uniformly distributed in the length $V$, the survival probability for the given trajectory

\footnotetext{
*Electronic address: lucian.anton@infim.ro

$\dagger$ Electronic address: richardb@theory.ph.man.ac.uk

$\ddagger$ Electronic address: bray@theory.ph.man.ac.uk
} 
is $(1-R(t ; z) / V)^{N}$ which, in the continuum limit $N \rightarrow \infty, V \rightarrow \infty$, with $\rho=N / V=\operatorname{constant,~becomes~} \exp \{-\mu[z]\}$, where $\mu[z]=\rho R(t ; z)$.

The statistical properties of the $A$ particle are calculated by averaging over the trajectories, where each trajectory is weighted with its survival probability multiplied by its Brownian weight. In this way one obtains the action functional 9]

$$
S[z]=\frac{1}{4 D_{A}} \int_{0}^{t} d \tau\left(\frac{d z}{d \tau}\right)^{2}+\mu[z]
$$

which defines an effective dynamics for surviving $A$ particle trajectories 9].

Further progress requires an explicit expression for the functional $\mu[z]$. In ref. [9] it was shown that the constant trajectory, $z(t)=0$, gives the global minimum of $\mu[z]$, and furthermore a systematic expansion in $D_{A} / D_{B}$ was obtained for $\mu[z]$. To first order one obtains [9]

$$
\mu[z]=\lambda t^{1 / 2}+\frac{\lambda}{8 \pi D_{B}} \int_{0}^{t} \frac{d t_{1}}{\left(t-t_{1}\right)^{1 / 2}} \int_{0}^{t_{1}} \frac{d t_{2}}{t_{2}^{1 / 2}\left(t_{1}-t_{2}\right)^{3 / 2}}\left[z\left(t_{1}\right)-z\left(t_{2}\right)\right]^{2}+O\left(z^{4}\right),
$$

where $\lambda=(2 / \pi) \rho\left(4 \pi D_{B}\right)^{1 / 2}$.

In this paper we study the asymptotic properties of the spatial fluctuation of the $A$ particle, using the formalism described above in the quadratic approximation for $\mu[z]$ given by Eq. (2). The paper is laid out as follows. In section II we apply the formalism developed in refs. [9, 14] to compute the root-mean-square fluctuation of surviving trajectories, and show that it scales as $t^{1 / 4}$. In section III we adapt this formalism to compute the root-mean-square fluctuation at time $t / 2$ for surviving 'loop' trajectories, constrained to return to their starting points at time $t$. This second calculation is motivated by the fact that it involves a very different mathematical treatment, and it is not obvious that the same fluctuation exponent will be obtained. Indeed, we find that this second quantity scales with a larger exponent $1 / 3$, which seems at first sight counter to physical intuition. Numerical results presented in section III are, however, consistent with these two different predictions. Section IV is a summary and conclusion. Some technical details are relegated to an appendix.

\section{SPATIAL FLUCTUATIONS OF SURVIVING TRAJECTORIES}

The probability for the $A$ particle, starting from the origin and following a trajectory $z(t)$, to reach $x$ at time $t$ can be written as

$$
p(x, t)=\mathcal{N} \int_{z(0)=0}^{z(t)=x} \mathcal{D}[z(t)] \exp (-S[z]) .
$$

Since we are interested in the spatial fluctuations of the surviving trajectories we choose the normalization factor $\mathcal{N}$ such that

$$
\int d x p(x, t)=1
$$

We can extract the $x$-dependence of the functional integral, Eq. (3), by expanding the trajectories $z(t)$ around the 'classical path', $z_{c l}(t)$, which minimizes the action $S$. Since the action is a quadratic functional of $z(t)$, we have

$$
p(x, t)=\mathcal{N} \exp \left(-S\left[z_{c l} ; x, t\right]\right) \int_{z(0)=0}^{z(t)=0} \mathcal{D}[z(t)] \exp (-S[z])
$$

and the remaining functional integral, over the fluctuations around the classical path, can be absorbed into the normalization constant, as can the $z$-independent part of $\mu[z]$, see Eq. (2).

To compute the probability distribution $p(x, t)$, therefore, it is sufficient to find the classical path $z_{c l}$, i.e. the path of least action. The analysis is simplified by the introduction of a dimensionless action, $\tilde{S}$, through the substitutions $t_{1} \rightarrow t u, t_{2} \rightarrow t v, z(t) \rightarrow x \xi$ in Eq. (2). This gives

$$
\tilde{S} \equiv \frac{2 D_{A} t}{z^{2}} S=\frac{1}{2} \int_{0}^{1} d u \dot{\xi}(u)^{2}+\frac{1}{2} \gamma(t) \int_{0}^{1} \frac{d u}{\sqrt{1-u}} \int_{0}^{u} \frac{d v}{v^{1 / 2}(u-v)^{3 / 2}}(\xi(u)-\xi(v))^{2}
$$


where $\gamma=(\lambda / 2 \pi)\left(D_{A} / D_{B}\right) t^{1 / 2}=\left(\pi^{2} / 2\right) g(t)$ is a dimensionless constant, the final equality defining $g$. In what follows, it is important to bear in mind that $g \propto t^{1 / 2}$, and that we are interested in the large- $t$ behaviour of the theory.

The minimum action can be computed using the observation that any trajectory starting at $\xi(0)=0$ and ending at $\xi(1)=1$ can be written as a straight line connecting the end points plus a Fourier expansion for the deviation from this straight line,

$$
\xi(u)=u+\sum_{n \geq 1} a_{n} \sin (n \pi u)
$$

where the linear part $u$ can also be written as a Fourier sine series:

$$
u=1-\frac{2}{\pi} \sum_{n \geq 1} \frac{\sin (n \pi u)}{n} .
$$

With these substitutions we can express the action, Eq. [6], in terms of the matrix elements $A_{m n}$ introduced in [14],

$$
\begin{aligned}
A_{m n} & =\frac{1}{m n} \int_{0}^{1} \frac{d u}{\sqrt{1-u}} \int_{0}^{u} \frac{d v}{\sqrt{v}(u-v)^{3 / 2}} \\
& \times[\sin (n \pi u)-\sin (n \pi v)][\sin (m \pi u)-\sin (m \pi v)] .
\end{aligned}
$$

After a straightforward computation we obtain:

$$
\tilde{S}=\frac{1}{2}+g \sum_{m, n} A_{m n}-\pi g \sum_{m, n} A_{m n} n a_{n}+\frac{\pi^{2}}{4} \sum_{m, n}\left(n^{2} \delta_{m n}+g A_{m n} n m\right) a_{m} a_{n} .
$$

The classical path is obtained by minimizing this expression with respect to the coefficients $a_{n}$, which gives

$$
\tilde{a}_{n}=\frac{2}{\pi} g \sum_{m, q}\left(\frac{1}{I+g A}\right)_{n m} A_{m q}
$$

where we have made the rescaling $a_{n}=\tilde{a}_{n} / n$. Hence the minimum action can be expressed in terms of the matrix elements $A_{m n}$ :

$$
\begin{aligned}
\tilde{S}_{c l}=\frac{2 D_{A} t}{x^{2}} S_{c l} & =\frac{1}{2}+g \sum_{m n} A_{m n}-g^{2} \sum_{\substack{m, n \\
p, q}}\left(\frac{1}{I+g A}\right)_{n p} A_{p q} A_{m n} \\
& =\frac{1}{2}+g \sum_{m, n, q}\left(\frac{1}{I+g A}\right)_{m q} A_{q n} .
\end{aligned}
$$

The desired probability distribution $p(x, t)$ has the Gaussian form

$$
p(x, t) \propto \exp \left(-S_{c l}\right)=\exp \left(-\frac{\tilde{S}_{c l}}{2 D_{A} t} x^{2}\right)
$$

with variance $\left\langle x^{2}\right\rangle=D_{A} t / \tilde{S}_{c l}$. We emphasize at this point that $\tilde{S}_{c l}$ depends on time through $g$. Our next goal, therefore, is to determine the $g$-dependence, at large $g$, of the minimum action given by Eq. (12). To this end we employ a finite-size scaling method, by computing numerically the action $S_{c l}$ for an $A$ matrix truncated to size $N$, and analyzing the dependence of $\tilde{S}_{c l}$ on $g$ and $N$.

To motivate the particular finite-size scaling form we use, we first consider the extreme limits $g \rightarrow \infty$ and $g \rightarrow 0$ at fixed $N$. For $g \rightarrow \infty$, Eq. (12) becomes $\tilde{S}_{c l}=1 / 2+\sum_{n=1}^{N} 1=N+1 / 2$, while for $g \rightarrow 0$ we obtain $\tilde{S}_{c l}=$ $1 / 2+g \sum_{n, m=1}^{N} A_{m n}+O\left(g^{2}\right)$. From the definition (9) of $A_{m n}$ it is easy to show that the double sum converges to the value $\pi^{3} / 6$ for $N \rightarrow \infty$. Thus we can write $\tilde{S}_{c l}-1 / 2=H(g, N)$, where $H(g, N) \rightarrow N$ for $g \rightarrow \infty$ and $H(g, \infty) \rightarrow\left(\pi^{3} / 6\right) g$ for small $g$. This suggests the finite-size scaling form $\tilde{S}_{c l}-1 / 2=N h(g / N)$, where $h(x) \sim x$ for small $x$ so that the $N$-dependence drops out as $N \rightarrow \infty$.

A scaling plot, testing this form, is presented in Figure 1 for a range of values of $g$ and $N$. The data collapse is excellent (the data sets are virtually indistinguishable) confirming the validity of the assumed scaling form. We conclude that, in the limit $N \rightarrow \infty, \tilde{S}_{c l} \sim g \sim t^{1 / 2}$, implying, via Eq. (13), that

$$
\left\langle z^{2}(t)\right\rangle \propto \rho^{-1}\left(D_{B} t\right)^{1 / 2} .
$$

Note that $\left\langle z^{2}(t)\right\rangle$ is independent of $D_{A}$ at leading order. 


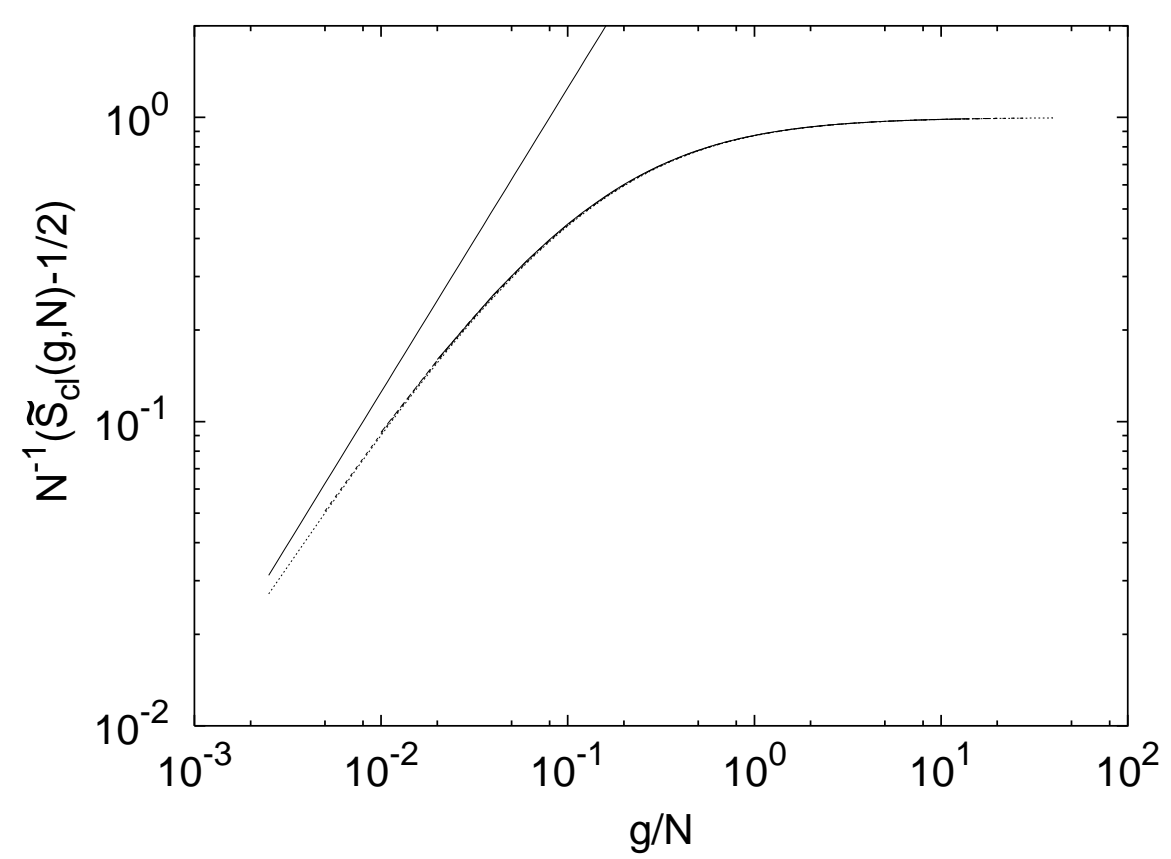

FIG. 1: Scaling of the $g$-dependent part of the dimensionless extremal action for free trajectories, for $N=50,100,200,400$. The continuous straight line with slope 1 is a guide to the eye. It indicates the expected asymptotic slope of the data for $g / N \ll 1$.

\section{FLUCTUATIONS OF CLOSED LOOPS}

We turn now to a discussion of the spatial fluctuations of closed loops, i.e. trajectories that return to the origin at time $t$. One might naively expect that the root-mean-square displacement of the trajectory at, say, its mid-point $t / 2$, would also scale as $t^{1 / 4}$. The mathematics of this calculation is, however, rather different and we shall see that the final result is different as well.

The probability distribution of the displacement $x$ of such a trajectory at time $t / 2$ is given by

$$
p^{(l o o p)}(x, t / 2)=\mathcal{N} \int_{z(0)=0}^{z(t)=0} \mathcal{D}[z(t)] \delta(x-z(t / 2)) e^{-S[z]} .
$$

Applying the standard 'exponentiation' of the delta function via an auxiliary integration, representing $z$ by a Fourier sine series on the interval $(0, t)$, and performing the resulting Gaussian integrals we obtain, after some straightforward algebra,

$$
p^{(l o o p)}(x, t / 2) \propto \exp \left[-\frac{x^{2}}{2 D_{A} t} F(g)\right]
$$

where

$$
F(g)=\frac{\pi^{2}}{4}\left[\sum_{m, n}\left(\frac{1}{1+g A}\right)_{m n} \frac{\sin (\pi m / 2) \sin (\pi n / 2)}{m n}\right]^{-1}
$$

It follows that

$$
\left\langle x^{2}(t / 2)\right\rangle_{\text {loops }}=\frac{D_{A} t}{F(g)}=\frac{4 D_{A} t}{\pi^{2}} \sum_{m, n=1}^{\infty}\left(\frac{1}{1+g A}\right)_{m n} \frac{\sin (\pi m / 2) \sin (\pi n / 2)}{m n} .
$$

Note the very different character of the calculations of $p(x, t)$ and $p^{(l o o p)}(x, t / 2)$. The former is completely determined by the classical path connecting the spacetime points $(0,0)$ and $(x, t)$. The fluctuations around this path are 


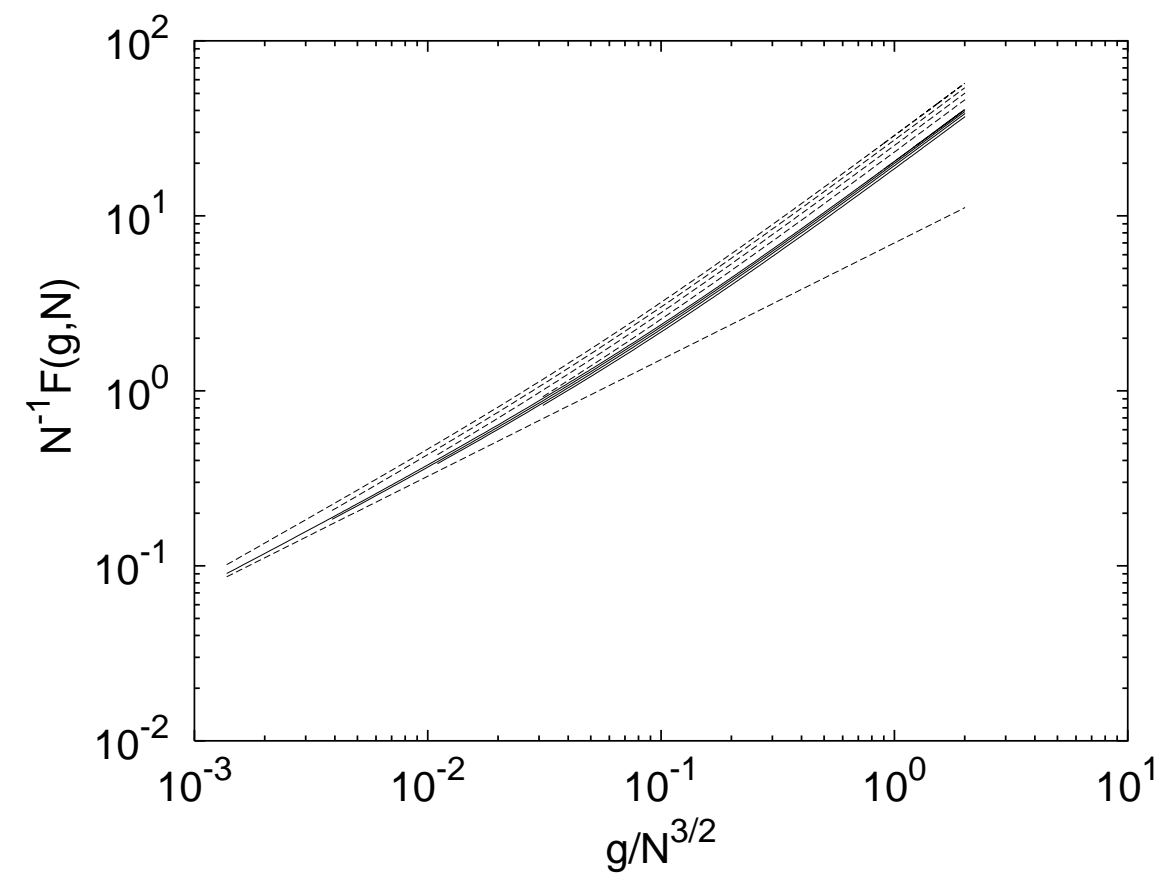

FIG. 2: Scaling of the function $F(g, N)$ (see text) for closed trajectories at time $t / 2$, for $N=50,100,200$, 400: continuous curves - full problem; broken curves - diagonal approximation. The straight line with slope $2 / 3$ is a guide to the eye. It represents the expected asymptotic slope of the data for $g / N^{3 / 2} \ll 1$.

not important for the calculation of $p(x, t)$ since they give an $x$-independent contribution, i.e. they only the affect the normalization of $p(x, t)$, which can in any case be fixed by hand a posteriori. By contrast, the calculation of $p^{(l o o p)}(x, t / 2)$, over paths connecting the spacetime points $(0,0)$ and $(0, t)$, is entirely concerned with fluctuations we need to explicitly integrate out the fluctuations to determine $p^{(l o o p)}(x, t / 2)$.

To determine $\left\langle x^{2}(t / 2)\right\rangle_{\text {loops }}$ from Eq. (16) we need to determine the form of the function $F(g)$ for large $g$. Again we can exploit a numerical finite-size scaling analysis. In view of the considerations in the preceding paragraph, we expect a rather different finite-size scaling form for the function $F(g, N)$, obtained from truncating the matrix A to size $N$, than we found for $\tilde{S}(g, N)$. In this context it is helpful to recall the calculation [14] of the subleading term in the $A$-particle survival probability, which is also a fluctuation-dominated effect. In that calculation it was found that approximating the $A$ matrix by its diagonal part gave a very good guide to the finite-size scaling behaviour. Using that approach here, we are led to consider the function

$$
F_{\text {diag }}(g, N)=\frac{\pi^{2}}{4}\left[\sum_{n=o d d}^{N}\left(\frac{1}{1+g A_{n n}}\right) \frac{1}{n^{2}}\right]^{-1} .
$$

For large $n, A_{n n} \propto n^{-3 / 2}$ (see the appendix) and, for large $N$ and $g$ it is clear that large $n$ dominate the sum, justifying the use of the large $n$ form of $A_{n n}$. Inserting this form in Eq. (19) we see that, for $N \rightarrow \infty, F_{\text {diag }} \sim g^{2 / 3} \propto t^{1 / 3}$ for large $g$, whence Eq. (16) gives $\left\langle x^{2}(t / 2)\right\rangle_{\text {loops }} \sim D_{A} t / g^{2 / 3} \propto \rho^{-2 / 3}\left(D_{A} D_{B}\right)^{1 / 3} t^{2 / 3}$ within the diagonal approximation.

Furthermore, the diagonal approximation (19) exhibits, for $g$ and $N$ both large, the scaling form $F_{\text {diag }}(g, N)=$ $g^{2 / 3} H\left(g / N^{3 / 2}\right)=N h\left(g / N^{3 / 2}\right)$. We adopt this same scaling form for the analysis of the full function $F(g, N)$, determined numerically. The results, together with the diagonal approximation, are shown in Figure 2. The data collapse is not as good as for the full set of paths (Fig. 1), with the data drifting to higher values for larger $N$. The same is true, however, of the diagonal data for which we know by explicit calculation that the assumed asymptotic scaling form is correct. Indeed, the full function $F(g, N)$ appears to be converging faster with increasing $N$ than the diagonal approximation to it. We are therefore confident that the assumed scaling is asymptotically correct and, as a consequence, $\sqrt{\left\langle x^{2}(t / 2)\right\rangle_{\text {loops }}} \sim t^{1 / 3}$.

An intriguing aspect of this result is that a subset of $A$-particle trajectories have spatial fluctuations scaling with a larger exponent $\left(\left\langle x^{2}\right\rangle_{\text {loops }}^{1 / 2} \sim t^{1 / 3}\right)$ than the whole set of trajectories $\left(\left\langle x^{2}\right\rangle^{1 / 2} \sim t^{1 / 4}\right)$. While this result seems at first sight counterintuitive, the numerical scaling analysis strongly suggests that it is correct. Further supporting data will 
be presented in the following section.

It is worth noting that, in this context, there is nothing special about the fluctuations of the mid-point of a closed path. For example, we can consider the mean square fluctuation, ${\overline{\left\langle x^{2}\right\rangle_{\text {loops }}}}=t^{-1} \int_{0}^{t} d t^{\prime}\left\langle x^{2}\left(t^{\prime}\right)\right\rangle_{\text {loops }}$, along the path. Using the usual Fourier expansion one readily obtains

$$
{\overline{\left\langle x^{2}\right\rangle_{\text {loops }}}}=\frac{2 D_{A} t}{\pi^{2}} \sum_{n=1}^{\infty} \frac{1}{n^{2}}\left(\frac{1}{1+g A}\right)_{n n} .
$$

This quantity will also scale as $t^{2 / 3}$ (i.e. the root-mean square fluctuation grows as $t^{1 / 3}$ ) as can be checked explicitly within the diagonal approximation. As a further twist, we can consider the fluctuations at time $t / 2$, or the root-mean square fluctuation over the whole path, of all trajectories that survive until time $t$. These both scale at $t^{1 / 3}$ in contrast to the fluctuation of the end-point of the path which, as we have seen, grows as $t^{1 / 4}$.

That the fluctuations of the trajectory at time $t / 2$, for paths which survive to time $t$, could be larger than the fluctuations at time $t / 2$ of paths which survive till time $t / 2$ has the following intuitive explanation. Trajectories which survive for a long time arise predominantly from initial configurations of the $B$ particles in which the $A$ particle is initially in a large region devoid of $B$ particles. If the $A$ particle is to survive until time $t$, this region is larger than if it only has to survive until time $t / 2$, so the fluctuations in its position at time $t / 2$ are expected to be larger in the former case than in the latter.

\section{NUMERICAL SIMULATIONS}

Numerical simulation data were obtained for both the unconstrained average $\left\langle z^{2}(t)\right\rangle^{1 / 2}$ and the average $\left\langle z^{2}(t / 2)\right\rangle_{\text {loops }}$ corresponding to surviving trajectories that return to the origin at time $t$. The data were generated using the lattice-based algorithm of Mehra and Grassberger [3, 12], which is readily adapted to the constrained case. The basic algorithm calculates the survival probability, averaged over all $B$-particle initial conditions and trajectories, of a given $A$-particle trajectory. Final results are obtained by averaging over a large number (typically $10^{5}$ ) of $A$ particle trajectories randomly generated from the $2^{t}$ possible paths of length $t$. In the modified algorithm, $A$-particle trajectories are generated dynamically. At a given time, the next step is taken to the left with a probability equal to the number of paths that reach the origin at time $t$ ('return paths'), given that the first step is to the left, divided by the total number of return paths from that point. This procedure generates the ensemble of closed paths with the required weights.

The parameter values used were $D_{A}=D_{B}=1 / 2$ and $\rho=1 / 2$. In both cases we divide the root-mean-square value of the displacement by $t^{1 / 4}$ and by $t^{1 / 3}$ to test the different predictions for the two types of average. The data are presented in Figures 3 and 4. For ease of comparison, we have defined the end-time of the unconstrained paths to be $t / 2$, i.e. all data are taken at the same real time.

Looking at Figure 3, we see that the closed-path (loop) data show an increasing trend with time $t$ consistent with an exponent larger that 1/4. A linear fit on a $\log$-log plot gives an exponent $\phi \simeq 0.33$, consistent with the predicted value of $1 / 3$. The unconstrained data also show an increasing trend, but there is a suggestion that the slope is decreasing at later times - the prediction $\phi=1 / 4$ requires that these data eventually reach a plateau as $t \rightarrow \infty$. A linear fit, on a $\log -\log$ plot, gives $\phi \simeq 0.30$ over this range. The companion Figure 4 , in which the data are scaled by $t^{1 / 3}$, tells a similar story. The closed-path data are consistent with $\phi=1 / 3$, but the data including all paths show a clear decrease with time, indicative of a smaller exponent.

To explore this behaviour in more detail, we have computed a time-dependent effective exponent, $\phi(t)$. For the computation we use the full data set, of which only a small subset (of the 'all paths' data) is displayed in Figs. 3 and 4. The total time interval is divided into consecutive bins of size $d t_{i}$. The average value of $\left\langle z^{2}(t)\right\rangle^{1 / 2}$ is computed in each bin to smooth the data prior to taking a numerical derivative. The effective exponent, $\phi\left(t_{i}\right)$, at time $t_{i}$ is approximated by the difference between the logarithms of the average value for bins $i$ and $i+1$ divided by the difference of the logarithms of the average positions of bins $i$ and $i+1$. The result of this procedure is shown in Fig. 5. The effective exponent is noisy, but is clearly decreasing with increasing time in a manner consistent with the predicted limiting value of $1 / 4$. The equivalent data for the closed paths is also shown. In this case, however, there are no more data available beyond that shown in Figures 3 and 4 - the simulations are computationally expensive due to the requirement that the paths return to the origin at time $t$. As a result, each bin contains a single data point, and the effective exponent is necessarily very noisy, but consistent with $\phi=1 / 3$. 


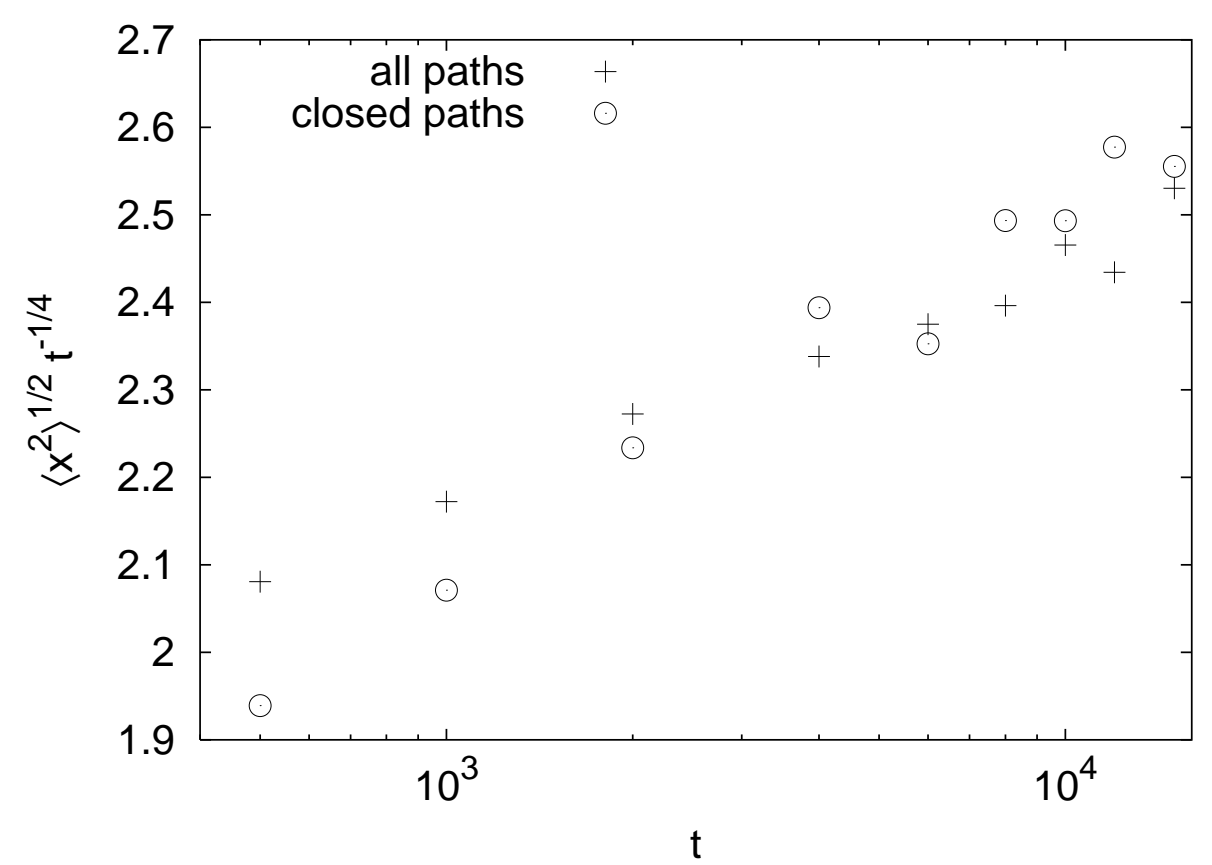

FIG. 3: Root mean square displacement at time $t / 2$, divided by $t^{1 / 4}$, for all paths and for closed paths that survive till time $t$.

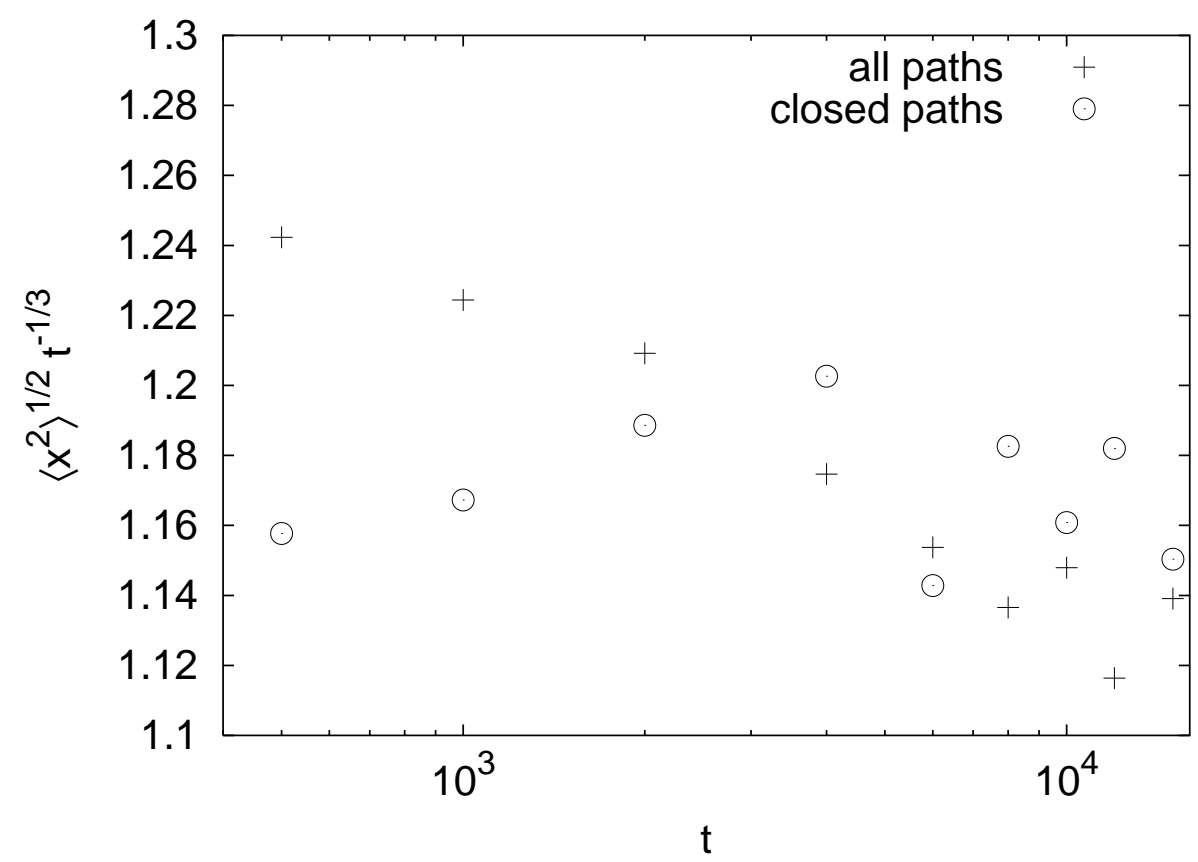

FIG. 4: Root mean square displacement at time $t / 2$, divided by $t^{1 / 3}$, for all paths and for closed paths that survive till time $t$.

\section{CONCLUSION}

We have studied the scaling properties of the spatial fluctuations of a diffusing particle interacting with diffusing traps, using the effective action arising from eliminating the trap degrees of freedom. The presence of the trapping process renders the fluctuations in the position of surviving particles subdiffusive, $\left\langle z^{2}(t)\right\rangle^{1 / 2} \propto t^{1 / 4}$, with an amplitude 


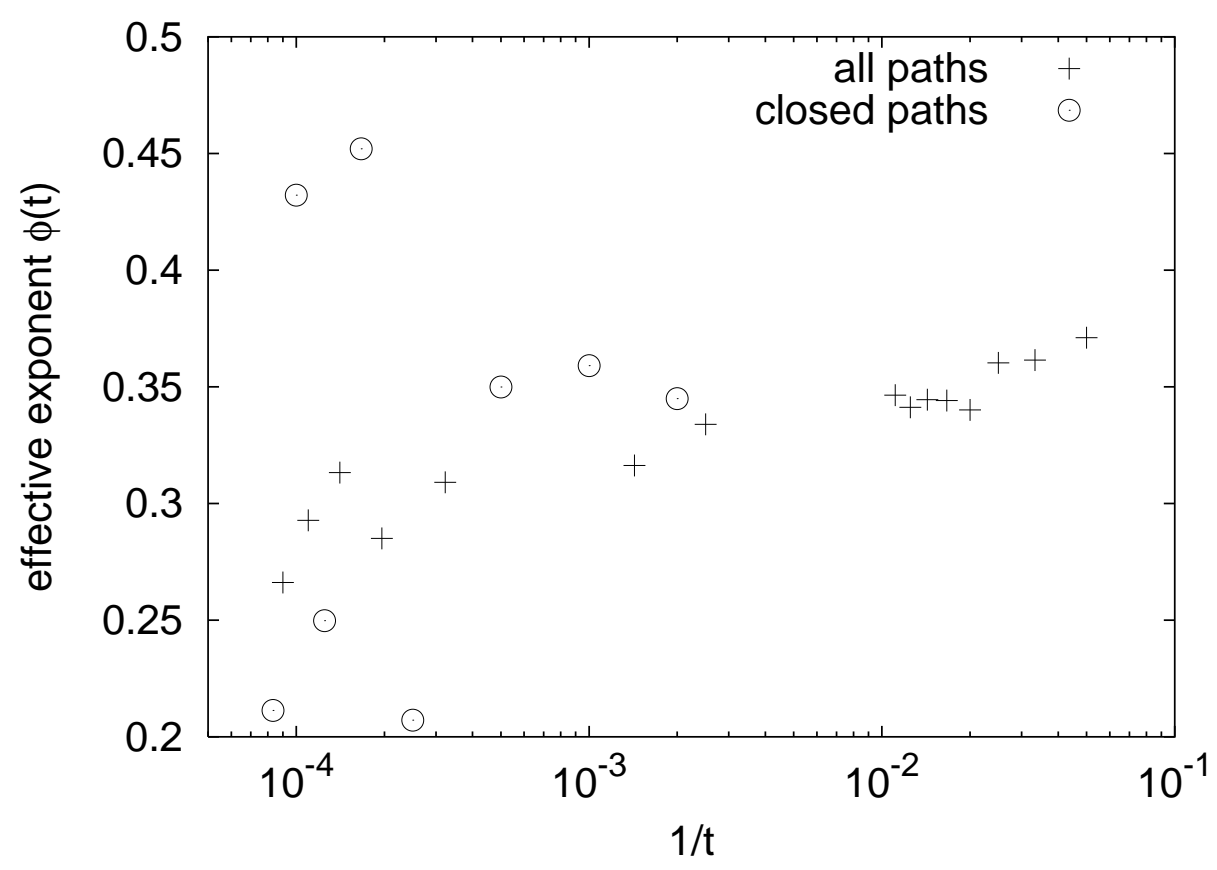

FIG. 5: Effective exponent $\phi(t)$ for all paths and for closed paths, plotted against $1 / t$.

that is independent of the $A$-particle diffusion constant, $D_{A}$. We have also found that the fluctuations at 'half-time' of surviving trajectories that return to the origin at time $t$ scale with a different exponent $\left\langle z^{2}(t / 2)\right\rangle_{(\text {loops })}^{1 / 2} \propto t^{1 / 3}$. As a cautionary note we recall that both of these results were obtained by using the quadratic approximation to the action functional, which is strictly valid only in the limit $D_{A} \ll D_{B}$. It remains an open question to what extent corrections to the quadratic approximation can modify the results for general values of $D_{A} / D_{B}$. In view of the reasonable qualitative agreement of the theory with numerical data for the case $D_{A}=D_{B}$, however, we expect any modifications to be quantitative rather than qualitative.

\section{Acknowledgements}

LA acknowledges support from the European Community Marie Curie Fellowship scheme under contract No. HPMF-CT-2002-01910. RAB acknowledges support from EPSRC under grant GR/R44768.

[1] See, e.g., S. Redner in Nonequilibrium Statistical Mechanics in One Dimension, edited by V. Privman (Cambridge University Press, Cambridge, 1997), and references therein.

[2] A. J. Bray and R. A. Blythe, Phys. Rev. Lett. 89 150601, (2002).

[3] R. A. Blythe and A. J. Bray, Phys. Rev. E 67, 041101 (2003).

[4] R. A. Blythe and A. J. Bray, J. Phys. A 35, 10503 (2002).

[5] G. Oshanin, O. Benichou, M.Coppey and M. Moreau, Phys. Rev. E 66, 060101 (2002).

[6] M. Moreau, G. Oshanin, O. Benichou and M. Coppey, Phys. Rev. E 67, 045104 (2003).

[7] M. Moreau, G. Oshanin, O. Benichou and M. Coppey, Physica A 327, 99 (2003).

[8] M. Moreau, G. Oshanin, O. Benichou and M. Coppey, Phys. Rev. E 69, 046101 (2004).

[9] A. J. Bray, S. N. Majumdar and R. A. Blythe, Phys. Rev. E 67, 060102 (2003).

[10] S. N. Majumdar and A.J. Bray, Phys. Rev. E 68, 045101 (2003).

[11] S. B. Yuste and L. Acedo, Physica A 336, 334-346 (2004).

[12] V. Mehra V and P. Grassberger, Phys. Rev. E 65050101 (2002).

[13] M. Bramson and J. L. Lebowitz, Phys. Rev. Lett. 61, 2397 (1988).

[14] L. Anton and A. J. Bray, J. Phys. A 37, 8407(2004). 


\section{APPENDIX A: THE MATRIX $A$}

The matrix element $A_{m n}$ is proportional to the double integral

$$
I_{m n}=m n A_{m n}=\int_{0}^{1} \frac{d x}{\sqrt{1-x}} \int_{0}^{x} \frac{d y}{\sqrt{y}(x-y)^{3 / 2}}[\sin (n \pi x)-\sin (n \pi y)][\sin (m \pi x)-\sin (m \pi y)]
$$

which, by the variable substitutions $z=x-y, s=y$ and standard manipulations of the trigonometric functions can be reduced to a sum of one-dimensional integrals,

$$
\begin{aligned}
I_{m n} & =2 \pi \cos \left(\frac{\pi(m-n)}{2}\right) \int_{0}^{1} \frac{d z}{z^{3 / 2}} \sin \left(\frac{n \pi z}{2}\right)\left(\sin \frac{m \pi z}{2}\right) J_{0}\left(\frac{\pi}{2}(m-n)(1-z)\right) \\
& +2 \pi \cos \left(\frac{\pi(m+n)}{2}\right) \int_{0}^{1} \frac{d z}{z^{3 / 2}} \sin \left(\frac{n \pi z}{2}\right) \sin \left(\frac{m \pi z}{2}\right) J_{0}\left(\frac{\pi}{2}(m+n)(1-z)\right),
\end{aligned}
$$

where $J_{0}(z)$ is the Bessel function of the first kind. The fact that $I_{m n}=0$ if $m+n=2 p+1$ can be obtained directly from Eq. A1 on noticing that the kernel is symmetric on reflection about the line $y=1-x$.

For the case $m=n$, the dominant large- $n$ contribution comes from the first integral:

$$
2 \pi \int_{0}^{1} \frac{d z}{z^{3 / 2}} \sin ^{2}\left(\frac{n \pi z}{2}\right) \approx 2 \pi \sqrt{n} \int_{0}^{\infty} \frac{d z}{z^{3 / 2}} \sin ^{2}\left(\frac{\pi z}{2}\right)=\sqrt{2} \pi^{2} n^{1 / 2}
$$

More elaborate is the calculation of the asymptotic behaviour for $I_{m n}$ at large $n$ with $m$ fixed and large. We do the same substitutions, $z=x-y, s=y$, in order to isolate the most singular part in Eq. A1. Then we integrate once by parts with respect to $z$ such that the singularity in $z$ becomes integrable: $z^{-3 / 2} \rightarrow z^{-1 / 2}$. In the next step we transform the products of trigonometric function into sums to give

$$
\begin{aligned}
\frac{1}{\pi} I_{n m} & =-(n-m) J_{n, n+m}-(n-m) J_{n-m, n-m}+(n+m) J_{n+m, n+m}+(n+m) J_{n, n-m} \\
& -(n+m) K_{n, n+m}-(n-m) K_{n-m, n-m}+(n+m) K_{n+m, n+m}+(n-m) K_{n, n-m},
\end{aligned}
$$

where the integrals $J_{n, m}$ and $K_{n, m}$ are given by

$$
\begin{aligned}
J_{n, m} & =\int_{0}^{1} \frac{d z}{z^{1 / 2}} \int_{0}^{1} \frac{d s}{s^{1 / 2}(1-s)^{1 / 2}} \sin \pi(n z+m(1-z) s) \\
K_{n, m} & =\int_{0}^{1} \frac{d z}{z^{1 / 2}} \int_{0}^{1} \frac{d s(1-2 s)}{s^{1 / 2}(1-s)^{1 / 2}} \sin \pi(n z+m(1-z) s) .
\end{aligned}
$$

Now we have to calculate these integrals to leading order in the $n \gg 1, m \gg 1$ and $n \gg m$. We show in detail the computation for $J_{n, n+m}$. We deform the integral from $(0,1)$ to two parallel lines of the form $0 \rightarrow \pm i \infty$ and $1 \pm i \infty \rightarrow 1$, the sign being chosen such that the intermediate segment $\pm i \infty \rightarrow 1 \pm i \infty$ vanishes. This gives

$$
\begin{aligned}
J_{n, n+m} & =\Im \int_{0}^{1} \frac{d z}{z^{1 / 2}} \int_{0}^{1} \frac{d s}{s^{1 / 2}(1-s)^{1 / 2}} e^{i \pi(n z+(n+m)(1-z) s)} \\
& =\Im \int_{0}^{1} \frac{d z}{z^{1 / 2}} e^{i \pi n z}\left[\int_{0}^{\infty} \frac{i d s}{\sqrt{i} s^{1 / 2}(1-i s)^{1 / 2}} e^{-\pi(n+m)(1-z) s}\right. \\
& -e^{i \pi \pi(n+m)(1-z)} \int_{0}^{\infty} \frac{i d s}{\left.\sqrt{-i s^{1 / 2}(1+i s)^{1 / 2}} e^{-\pi(n+m)(1-z) s}\right]} \\
& \approx \Im\left[\frac{\sqrt{i}}{\sqrt{(n+m)}} \int_{0}^{1} \frac{d z e^{i \pi n z}}{\sqrt{z(1-z)}}-\frac{e^{i \pi(n+m)}}{\sqrt{i} \sqrt{(n+m)}} \int_{0}^{1} \frac{d z e^{-i \pi m z}}{\sqrt{z(1-z)}}\right] \\
& \approx \Im\left[\frac{\sqrt{i}}{\sqrt{(n+m)}}\left(\frac{\sqrt{i}}{\sqrt{n}}-\frac{e^{i \pi n}}{\sqrt{i} \sqrt{n}}\right)-\frac{e^{i \pi(n+m)}}{\sqrt{i} \sqrt{(n+m)}}\left(\frac{\sqrt{-i}}{\sqrt{m}}+\frac{\sqrt{i} e^{-i \pi m}}{\sqrt{m}}\right)\right] \\
& =\frac{1}{\sqrt{n+m}}\left(\frac{1}{\sqrt{n}}-\frac{\cos \pi(n+m)}{\sqrt{m}}\right)
\end{aligned}
$$




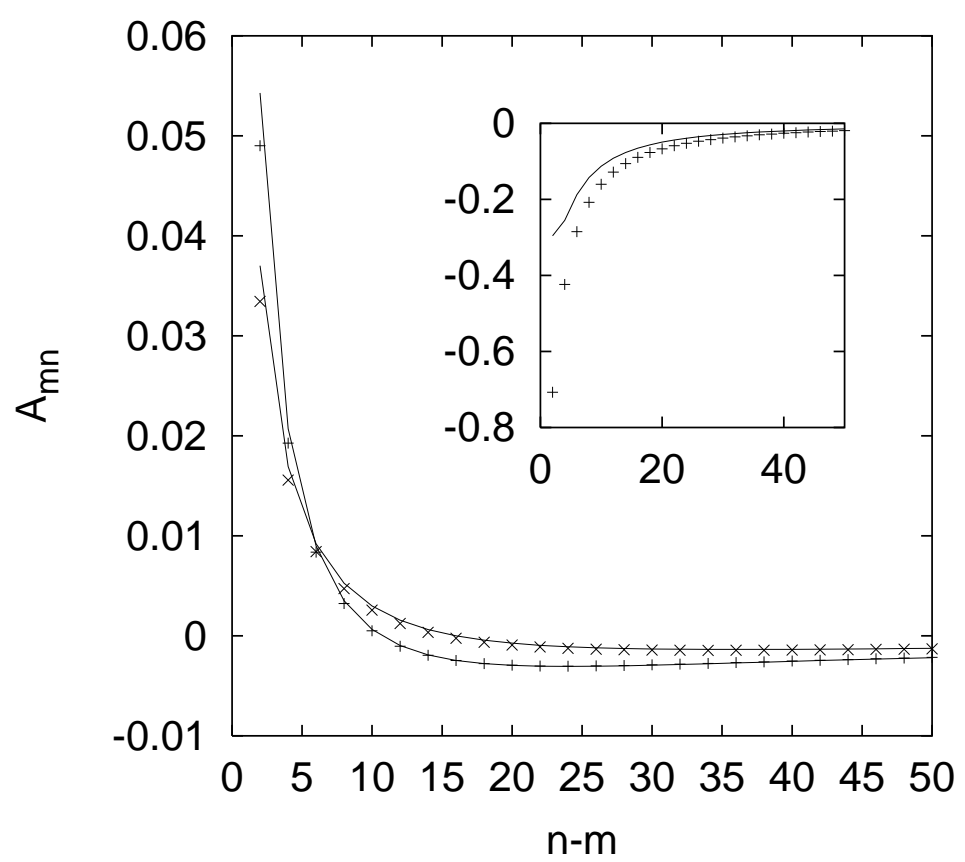

FIG. 6: $A_{m n}$ as s function of $n$ at $m=50,55$ starting from the main diagonal of the matrix. In the inset $A_{1 n}$ is compared with the asymptotic result. The symbols are the from the numerical integration of Eq. (A1), the lines are the asymptotic approximation, Eq. A13.

where we have used, to leading order,

$$
\int_{0}^{\infty} \frac{d x}{x^{1 / 2}} Q(x) e^{-k z} \approx Q(0) \frac{\sqrt{\pi}}{\sqrt{k}}
$$

when $Q(0)$ exists and $k \gg 1$.

Special attention must be paid to the point $z=1$ in the integral over $s$, as the argument in the exponential is not large when $z \rightarrow 1$ with $m, n$ fixed. However, one can show that this region gives a subleading contribution.

Applying the same procedure to the remaining integrals, we find that the contribution of $J_{n-m, n-m}, J_{n+m, n+m}$ and $K_{n-m, n-m}, K_{n+m, n+m}$ cancel each other to leading order and we finally obtain the closed-form expression

$$
A_{m n} \approx \frac{2 \pi}{m n}\left(\frac{\sqrt{n}}{\sqrt{n-m}}-\frac{\sqrt{m}+\sqrt{n}}{\sqrt{m+n}}\right)
$$

for $m \gg 1, n \gg 1$ and $n \gg m$. It is worth noting that the above result is a good approximation for any value of $m, n$ outside the main diagonal as one can see from Fig. 6] This fact is useful in numerical computations since the integrals that define $A_{m n}$ have strongly oscillating integrands for large $m, n$. 